\title{
Usability requirements for architectural analysis tool to support CBD
}

\begin{abstract}
Component-based development is an architecture-centric process that relies on the integration of pre-fabricated software components. These are often blackbox components whose functionality and configuration may not match the ñdealò system architecture. Systematic architectural analysis can help ensure that risks resulting from architectural adaptations and trade-offs do not adversely affect critical system qualities (e.g. performance, security and modifiability). However, architectural analysis is a complex activity that involves planning, analysis, negotiation and assessment of large amounts of interrelated, often conflicting information. Good tool support is therefore essential for effective architectural analysis. However, the success of a tool depended not only on powerful analysis methods but also on the quality of the toolset's usability. This paper presents functional and non-functional requirements of the toolôs user interface, and discusses the HCI design principles and guidelines in designing a high-quality user interface that would allow developers to parse, analyse and modify architecture specification easily and effectively.
\end{abstract}

Keyword: Tool; Architecture analysis; Usability; User interface design 